\title{
Intricate Analysis of Potential Materials for Sustainable Product Development
}

\author{
Sachin Kumar ${ }^{1}$, Anchit Goel ${ }^{2}$ and Gaurav Kumar Singh ${ }^{1}$
}

\begin{abstract}
Raw materials are scarce and are exponentially depleting. Material usage and product disposal both lead to improvidence of raw ingredients for products. We use materials for manufacturing new products while simultaneously throwing away used non-biodegradable products which pollute the environment. The world of designing and manufacturing needs to use new material(s) which can be selected using the methodology proposed in the research text. To select the best material for a product, its environmental impact, cost and properties that decide the quality of a product are plotted in a 3 dimensional space. Now the threshold limit of quality defining properties is obtained through experimentation. Cutoff cost is decided through market research and then environmental impact is minimized. The material beyond the cutoff planes is rejected and the best among the remaining materials is selected. The product developed using this method for material selection will contain the negative environmental impact during manufacture, use and expenditure of used products and thus helping sustainable development.
\end{abstract}

Keywords: Sustainability, Life Cycle Analysis, Material selection, Cost optimization, Environmental Repercussion, Mechanical properties

\section{Introduction}

Climate change is one of the major problems faced by all living creatures. For past decades the climate has been changing drastically. And due to this change, the lives of trillions of living species on earth are in danger. Even the extinction of species could result in a misbalance of the whole eco-system. All this is due to the pollution caused by the products that we manufacture and use in our daily lives. These products cause pollution during manufacturing and even after their use. The increase in population results in increasing demand for products for daily use. The increase in products manufactured per year increases pollution. Used products that don't degrade cause large heaps of waste which again pollutes the land.

Raw materials are exponentially depleting and the extraction of these raw materials is becoming difficult, costly and emissive. These emissions are harmful to the environment and all living species. Also, the better quality of the product would imply long term use and hence lesser waste/disposal. This decrease in lesser consumption of products would result in lesser manufacturing and raw material extraction. Hence better quality products will reduce the harmful effect on the environment and help in the conservation of crucial raw materials for future generations. Also, the product must have a low cost in order to sell out. A sustainable product has no use if it is costly as it won't sell out.

For the above reasons, a better way to select materials for a product is needed. For a product to be sustainable, material, design and manufacturing process selection are the major aspects that should be focused. Hence for sustainable product development, a 
simultaneous study of product's properties, cost, and environment effect during its life cycle are needed. In practical engineering, these aspects are evaluated separately and their effect on each other is not considered. Hence a new approach with a simultaneous complex study of these factors needs to be carried out for sustainable product development. This new approach evaluates the values of each factor considering cost, quality, and environmental impact separately and then a combined comparative study of these values for all potential materials is carried out to select the best among all.

\section{Generation of Standard Equations to Evaluate Sustainability of a Product}

The science of material selection involves the evaluation and comparison of many parameters. The most important among them is the cost of manufacturing and the quality of the product. Due to depleting resources and increasing population, a new parameter is added to make the process more sustainable. This new parameter represents the environmental effect of material during its life cycle. It can be represented in the form of cost to the environment. The value of this parameter represents the cost that could reverse the environmental impact during the materials life cycle. This is evaluated using Life Cycle Analysis (LCA). LCA involves emissions caused by product/material during its complete life cycle i.e. from the extraction of raw material to dispose of/recycle of material.

To represent the quality of the product, one or more specific properties of the product are considered. For example, a spacecraft material should be light and temperature resistant. So, the property that should be considered is the material's density and melting point. Hence the quality parameter for this product should be equivalent to the density of the material used to manufacture this product. The third parameter represents the cost of the manufacturing of the product. This includes raw material cost and manufacturing cost. Raw material cost includes the cost of extraction/recycling and transportation. Whereas manufacturing cost includes labor cost and power cost.

\subsection{Cost of product}

A traditional way to analyze the cost of the product is to simply add total money spent to manufacture a product. A cheaper product might cost very much in the long term. Products made of different materials have a different lifespan. So, the cost of owning a product for a specific period of time should depend not only on the cost of the product but also on the lifespan of the product. Hence the traditional method to analyze the total cost of the product is not fair to products made of different materials. Therefore, a new method is required to calculate the total cost of the product. The total cost of the product calculated should incorporate the lifespan of the product, recyclability ratio and recycling cost of one product. The lifespan of a product is actual time (in years) until the failure of the product. The expected value of lifespan can be predicted using analysis and simulation software for the design and each material. Recyclability ratio is the fraction of total material in a product that can be recycled. Recycling cost is the amount of money spent to recycle material from a used product. For the first cycle of the product, the cost of the product is a function of raw material cost per product and manufacturing cost per product only. Hence 
$C_{1}=R+M$

For further cycles of product, recyclability is also considered. Hence

$C_{2}=r+(1-\propto) \cdot R+M$

Therefore a combined equation to calculate the cost of the product for $\mathrm{N}$ years can be written as

$$
\begin{aligned}
& C=R+M+[r+(1-\propto) \cdot R+M] \cdot \frac{N-L}{L} \\
& C=\propto \cdot R-r+[r+(1-\propto) \cdot R+M] \cdot \frac{N}{L}
\end{aligned}
$$

Where

$\mathrm{C}_{1}$ is the cost of a product in its first lifespan

$\mathrm{C}_{2}$ is the cost of the first recycled product

$\mathrm{C}$ is the cost to keep a product in the market for $\mathrm{N}$ years

$\mathrm{R}$ is the cost of raw material used in a product

$\mathrm{M}$ is the manufacturing cost per product

$r$ is the cost to recycle one product

$\alpha$ is the recyclability ratio

$\mathrm{L}$ is the lifespan of a product

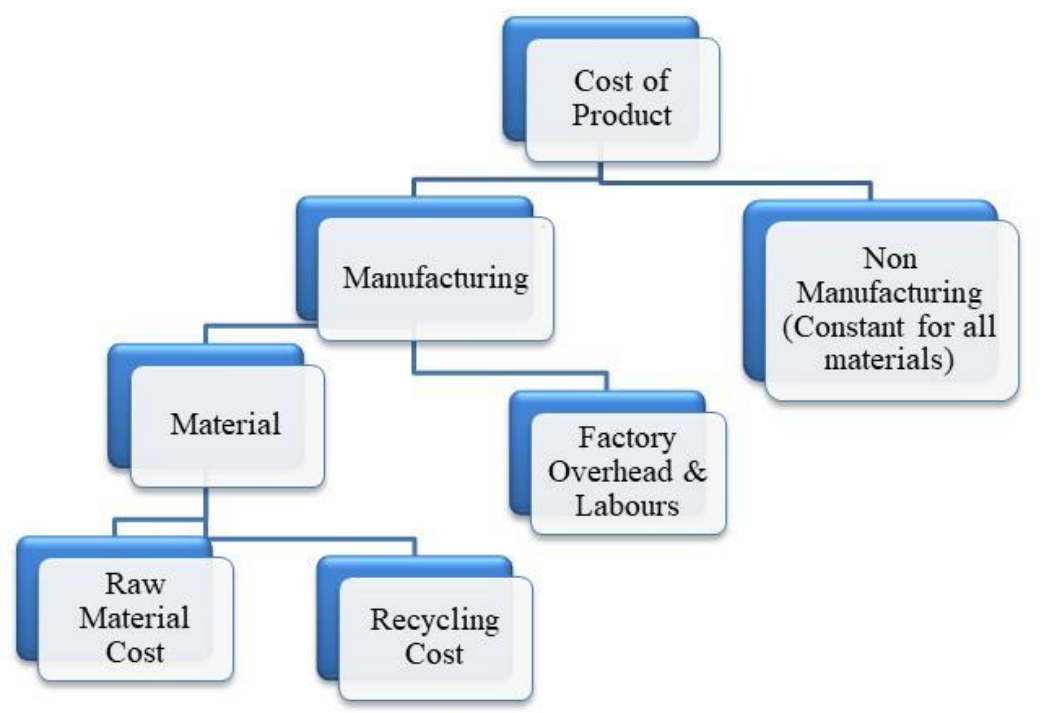

Fig1. Cost of product

\subsection{Properties of material}

Properties of material are also very important and considered equally important in the process of material selection. Properties of material are classified in two groups i.e. (a) properties that define the lifespan of the product and (b) properties that define the quality of the product in its lifespan. Group (a) properties are used to predict the lifespan of the product which is then used to calculate the cost of the product. Whereas, properties of the group (b) are directly used in the process of material selection. These properties are used to discard materials using cutoff for each. 


\section{Fig2. Properties of material}

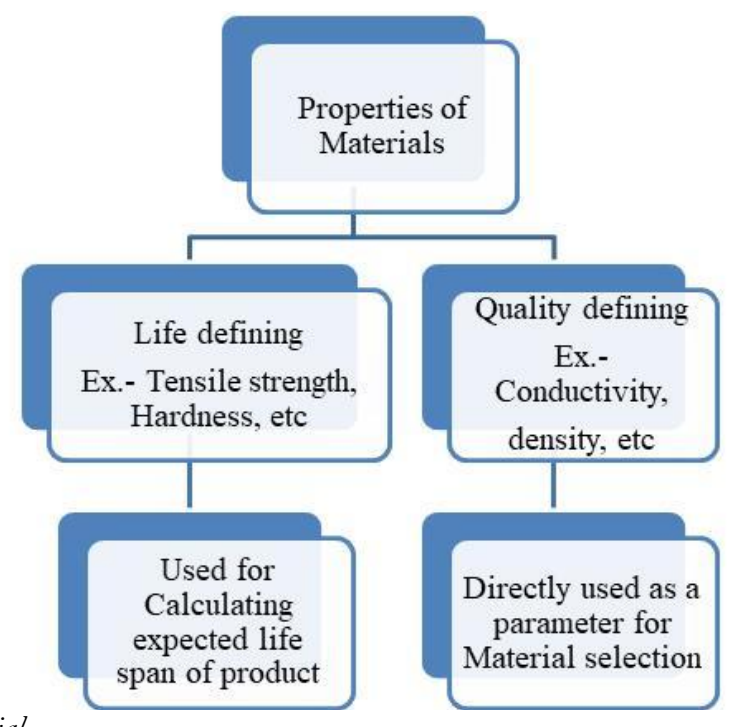

\subsection{Environmental impact of product}

Products can be very harmful to the environment and these environmental impacts are very difficult to recover from. Products are harmful to the environment at every stage in their entire life cycle. Hence it is most important to minimize these impacts. To minimize these impacts, careful selection of design, material, manufacturing process and recycling process is required. In the process of material selection, various calculations and estimations are required. These calculations are carried out using Life Cycle Analysis (LCA). LCA is used to analyze total emissions made during the complete life cycle of the product. The life cycle of the product involves the extraction of raw materials from their natural state, manufacturing processes, the complete lifespan of the product and recycling/dispose of the product.

During the extraction of raw material, different harmful gases and solid are emitted. Different materials have different quality and quantity of these emissions. Therefore different materials have different environmental impacts even before their use in a product. All materials have different mechanical properties, castability, and machinability. So, all materials consume different amounts of energy during the manufacturing phase. The energy consumed also has some environmental impact as not all of the energy is clean. So the higher the energy consumption, the higher is the environmental impact. The development of correlation for the environmental cost is similar to the equation generated for the cost of the product. Therefore, a final equation to calculate the environmental cost of the product can be written as,

$$
\begin{aligned}
& C_{e n v}=E+M_{e n v}+(1-\alpha) \cdot D+\left[r_{e n v}+(1-\alpha) \cdot E+M_{e n v}+(1-\alpha) \cdot D\right] \cdot \frac{N-L}{L} \\
& C_{e n v}=\propto \cdot E-r_{e n v}+\left[r_{e n v}+(1-\propto) \cdot E+M_{e n v}+(1-\propto) \cdot D\right] \cdot \frac{N}{L}
\end{aligned}
$$

Where,

$\mathrm{C}_{\text {env }}$ is the environmental cost of the product

$\mathrm{E}$ is the environmental cost during the extraction of raw material 
$\mathrm{M}_{\mathrm{env}}$ is the environmental cost during the manufacturing of the product

$\alpha$ is the recyclability factor

$r_{\text {env }}$ is the environmental cost during recycling

$\mathrm{D}$ is the cost to dispose of non-recyclable product material

$\mathrm{L}$ is the lifespan of the product

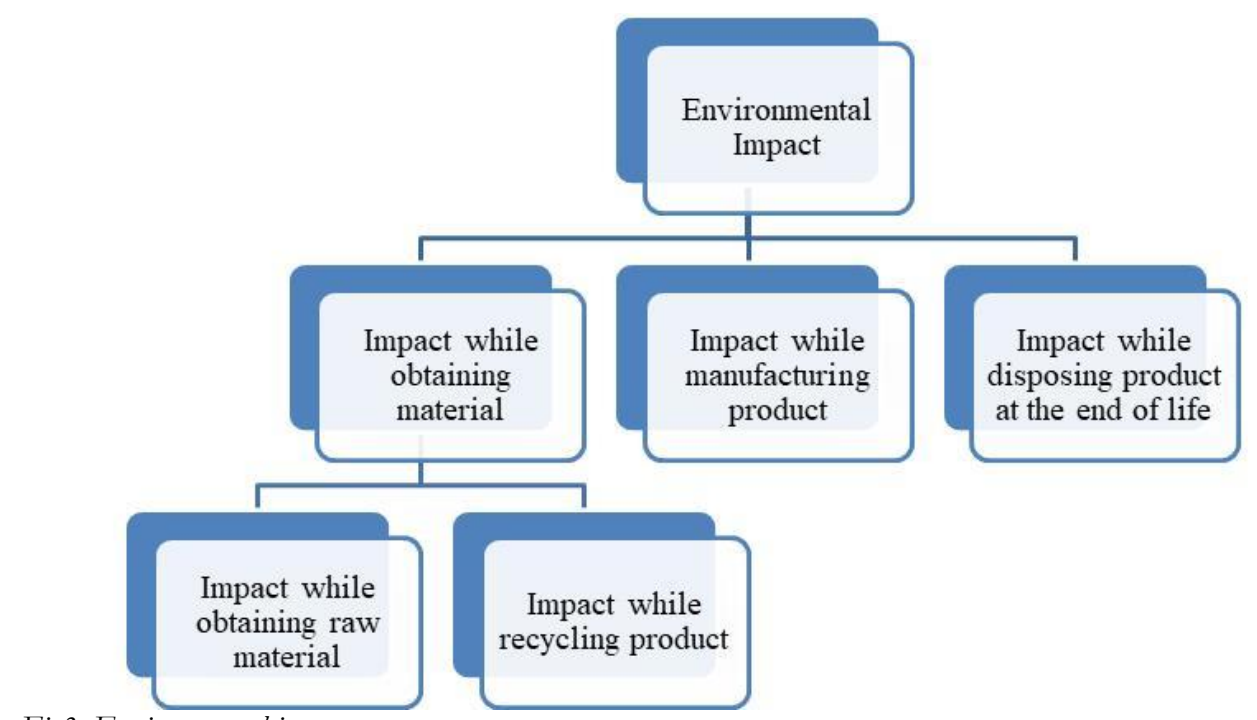

Fig3. Environmental impact

\section{Illustration of Methodology}

The sustainability parameters calculated using standard equations are used to select the best among potential materials for a product. All potential materials are plotted on a three-dimensional graph with each sustainability parameters on each axis of the graph. Materials might have different values of sustainability parameters depending on composition, temperature, etc, therefore the position of each potential material can be represented using a point, line or a solid on the three-dimensional graph.

Table 1: Potential materials, cost and density

\begin{tabular}{|l|c|c|}
\hline Material & Cost(USD per pound) & Density $\left(\mathrm{lb} /\right.$ in $\left.^{3}\right)$ \\
\hline Aluminum & 0.30 & 0.098 \\
\hline Iron/Steel & 0.24 & 0.284 \\
\hline Titanium & 30 & 0.161 \\
\hline Zinc & 1.05 & 0.258 \\
\hline Magnesium & 2.10 & 0.064 \\
\hline copper & 1.80 & 0.323 \\
\hline
\end{tabular}

Now the cutoff value of each sustainability parameter is used to discard material from the list of potential materials for the product. The cutoff value for the cost of the product is calculated with the help of market research based on demand and availability 
of the product in the market. The cutoff value of quality defining properties is obtained by performing experimental and analytical studies using different simulation and analysis software. Deciding cutoff values of environmental cost is the most difficult part. It depends on the environmental policies of the particular nation. The methodology is explained below using an example.

Ideal material for an aircraft skin would be light, cheap and one that causes less pollution. Potential materials for an aircraft are aluminum, steel, titanium, zinc, magnesium, and copper. Table 1 shows the cost and density of these materials. Each material among these has a different impact on the environment due to different emissions and energy consumption during extraction and manufacturing. The cost of the product is based on the amount of material used and manufacturing cost. The amount of material used would be different for each material as a fixed volume material is required but the density of each material is different. Also, simulation software like Ansys is used to calculate the expected life span of the plane. The property considered for the selection would be the density of the material.

Now to calculate environmental impact, emissions during extraction and energy consumption during manufacturing are considered. Also, recyclability of metals is considered. Using the generalized equation for each parameter, the values of each parameter are calculated and plotted on a $3 \mathrm{~d}$ plot. And different planes representing the cutoff values of each parameter are drawn. Materials outside the required are discarded.

\section{Summary and Conclusion}

Raw materials on our planet are limited but the population is not. Also, the extraction of rare raw materials causes more pollution. To avoid environmental pollution and scarcity of raw materials a new methodology for material selection is required that considers the cost and quality of the product as well as the environmental impact of the product for a specific material.

This research work presents a methodology for the selection of best material among potential materials. Three different parameters such as the cost of the product, properties of the product and environmental impact of a product in its life span have been considered for the selection of material. All potential materials were plotted on a three-dimensional space with each axis representing one parameter. Cutoff values of each parameter were decided based on simulations, market analysis and environmental policies of the particular nation. Materials outside these cutoff limits were discarded.

This research is just the beginning and does not present a solution to the problem specific to a product. Each product follows a different life cycle, extraction, and manufacturing process. Hence further research should be carried out to a specific field. Recycling and disposing of are also the processes that should be studied and further research should be carried out in these areas.

\section{References}

M. F. Ashby, Material Selection in Mechanical Design. 4th ed. Oxford: Butterworth-Hienemann; 2010. 
P. Stoffels, J. Kaspar, D Baehre, M. Vielhaber, Holistic material selection approach for more sustainable products, 2017

Y. Brechet, D. Bassetti, D. Landru, L. Salvo, Challenges in material and process selection, 2001

F. Giudice, G. La Rosa, A. Risitano, Material selection in the Life-Cycle Design process: a method to integrate mechanical and environmental performances in optimal choice, 2005

Lennart Y. Ljungberg, Material selection and design for development of sustainable products, 2007

Y. M. Deng, K. L. Edwards, The role of material identification and selection in engineering design, 2007

Rosy W. Chen, D. Navin-Chandra, T. Kurfess, F. Prinz, A systematic methodology of material selection with environmental consideration, 1994

J. Kaspar, M. Vielhaber, Cross-component systematic approach for lightweight and material oriented design, 2016

M. F. Ashby, Y. J. M. Brechet, D. Cebon, L. Salvo, Selection strategies for materials and processes, 2004

E. A. Starke, J. T. Staley, Application of modern aluminum alloys to aircraft, 1996 